\title{
Geometric Transition for a Class of Hyperbolic Operators with Double Characteristics
}

\author{
By Enrico BERNARDI and Antonio BOVE \\ Dipartimento di Matematica, Università di Bologna, Italy \\ (Communicated by Kiyosi ITô, M. J. A., April 12, 1995)
}

1. Introduction. The Cauchy problem in the $C^{\infty}$ category for hyperbolic operators with double characteristics has been studied by many authors under different geometrical perspectives. These results (see e.g. [15], [1] and the references therein) put in evidence the role of both the lower order terms and the symplectic geometry associated with the principal symbol at the double characteristic points.

The purpose of our work is the study of a case in which the set of double points $\Sigma_{2}$ can be written as the reunion of two nonempty subsets $\Sigma_{2, e}, \Sigma_{2, n e}$ i.e. the set of effectively hyperbolic double points and its $\Sigma_{2}$-complementary respectively. We say that in this case a "transition" occurs, or, roughly speaking, that a real eigenvalue of the fundamental matrix defined on $\sum_{2, e}$ vanishes on $\sum_{2, n e}$.

Trying to provide a unifying approach to the first of the open problems mentioned above, R. B. Melrose, [15], in 1984 formulated the following conjecture:

Conjecture. If the Cauchy problem for $P$ (having at most double characteristics) is well-posed in $\Omega_{0}$ then $s(\rho) / \lambda(\rho)$ is uniformly bounded in $\sum_{2, e} \cap$ $T^{*} V \backslash\{0\}$, for some neighborhood $V$ of $0 \in \boldsymbol{R}^{n+1}$, where

$$
\begin{aligned}
& s(\rho)=\left|\operatorname{Im} P_{m-1}^{s}(\rho)\right| \\
& \quad+\inf \left\{\left|\operatorname{Re} P_{m-1}^{s}(\rho)-s\right| ;|s| \leq \operatorname{Tr}^{+} F_{P_{m}}(\rho)\right\} .
\end{aligned}
$$
This conjecture, as it stands, has not been proved yet, however it gives a hint of what one should reasonably look for, when trying to formulate Levi conditions on the subprincipal symbol $P_{m-1}^{s}$.

The purpose of this paper is actually to understand a degenerate effectively hyperbolic case: more precisely we will show that the uniform boundedness of the ratio $s(\rho) / \lambda(\rho)$ may also serve as a sufficient condition for the wellposedness of the Cauchy problem, provided certain symplectically invariant geometric conditions are satisfied. Our Assumption (H4) is therefore a slightly restricted reformulation of Melrose Conjecture, and this is expected since we are dealing with a sufficiency result.

Even under the usual assumption that the principal symbol $P_{m}$ vanishes exactly of order two on $\Sigma_{2}$, one readily sees that the nature of the set $\Sigma_{2, n e}$ (henceforth denoted by $\Sigma_{2}^{\prime}$ ), the order of vanishing of $\lambda$ on $\Sigma_{2, n e}$ and the geometry of the transition from a symplectic point of view can be of a very wild type and may produce nontrivial situations.

Therefore we started assuming that $\Sigma_{2}$ is a smooth submanifold of $T^{*} \Omega \backslash\{0\}$. Furthermore in Section 3 below we collected a number of results serving as a symplectic classification of the transition cases that can possibly occur. In this framework Assumption (H2) isolates one of these cases.

More precisely (H2) takes a picture of $F_{P_{m}}(\rho)$ when $\rho$ is inside or outside $\Sigma_{2}^{\prime}$, avoiding for instance Jordan blocks of size 4 in the canonical form of $F_{P_{m}}(\rho), \rho \in \Sigma_{2}^{\prime}$ and precising that $0 \leq \lambda(\rho) \leq \operatorname{dist}_{\Sigma_{2}}^{2}\left(\rho, \Sigma_{2}^{\prime}\right)$, where dist denotes any geodesic distance of $\rho \in \Sigma_{2}$ from $\Sigma_{2}^{\prime}$; but this is not yet enough to prove an energy estimate eventually leading to existence and uniqueness. Indeed we recall that in the non degenerate effectively hyperbolic case (see e.g. Lemma 1.2.1 in [17]), denoting by $\Gamma_{P_{m}}(\rho)$ the hyperbolicity cone of the localized hyperbolic quadratic form $\sigma\left(X, F_{P_{m}}(\rho) X\right), " P_{m}$ is effectively hyperbolic at $\rho$ " if and only if " $\Gamma_{P_{m}}(\rho) \cap \operatorname{ran} F_{P_{m}}(\rho) \cap\left[\left(0, e_{0}\right)\right]^{\sigma}$ $\neq \emptyset$, where with $V^{\sigma}=\left\{z \in T_{\rho} T^{*} \Omega \mid \sigma(z, v)=\right.$ $0, \forall v \in V$ \} we define the dual with respect to the symplectic form $\sigma$ of a vector subspace $V$ of $T_{\rho} T^{*} \Omega$.

Therefore for an effectively hyperbolic operator it is always possible to find a time function $f$, i.e. a $C^{\infty}$ function vanishing on the double set of $P_{m}$, whose Hamilton vector field $H_{f}(\rho), \rho \in$ $\Sigma_{2}$ belongs to $\Gamma_{P_{m}}(\rho)$ (and for which in addition 
$\partial f / \partial \xi_{0}(\rho)=0$ holds). The usual strategy in this setting is to take advantage of this time function, as in [15] and [17], and use a suitable weight function in order to prove an energy estimate implying well-posedness.

On the other hand one quickly realizes that (see Section 3, example B) Assumption (H2) is too static to handle degenerate effectively hyperbolic cases. The existence of a "generalized" time function adapted to a "good" factorization of $\boldsymbol{P}_{m}$ is what we need here in order to take care of these difficulties. Roughly speaking we need a "real" time function in $\Sigma_{2} \backslash \Sigma_{2}^{\prime}$, whose existence is of course guaranteed by $(\mathrm{H} 2)$, such that $H_{f}(\rho)$, when $\rho \in \sum_{2} \rightarrow \bar{\rho} \in \sum_{2}^{\prime}$ has a smooth exit from $\Gamma_{P_{m}}(\rho)$ to $\left.\operatorname{ker} F_{P_{m}}(\bar{\rho})\right)$. The precise type of requests we are asking on the "time" function $f$ is written down explicitly in Assumption (H3). We would also like to point out that under the geometrical prescription of Hypothesis (H2) it is always possible to exhibit a good factorization of the principal symbol of $P$, according to Definition 2.1 in a way reminiscent of the well-known Ivrii factorization (see e. g. Ivrii [6]). The couple (H2), (H3) selects therefore a class of differential operators with double characteristics, whose fundamental matrix admits a non-negative real eigenvalue $\lambda(\rho)$, vanishing of order at least two on $\sum_{2}^{\prime}$, for which the uniform boundedness of the ratio $s(\rho) / \lambda(\rho)$ in Melrose conjecture proves to be the sufficient condition ensuring the correctness of the associated Cauchy problem.

2. Assumptions and the result. Let $P(x, D)$ be a differential operator of order $m$,

(2.1) $P(x, D)=P_{m}(x, D)+P_{m-1}(x, D)+\cdots$ where $P_{j}(x, D)$ denotes the homogeneous part of order $j$ of $P, j=0, \ldots, m$. We assume that $P$ is a differential operator with $C^{\infty}$ coefficients in the open set $\Omega \subset \boldsymbol{R}^{n+1}, 0 \in \Omega$. In this Section we list our assumptions on the operator $P$.

The first hypothesis is concerned with the intrinsic properties of the double characteristics of the principal symbol of $P$.

(H 1) ( i ) The principal symbol $P_{m}(x, \xi)$ of $P$ is hyperbolic with respect to the level surfaces of $x_{0}$.

(ii) The characteristic roots of $\xi_{0} \rightarrow P_{m}(x$, $\left.\xi_{0}, \xi^{\prime}\right)$ have multiplicity at most two; denoting by $\sum_{2}=\{(x, \xi) \in$ $T^{*} \Omega \backslash\{0\} \mid P_{m}(x, \xi)=0, d P_{m}(x, \xi)=0$, $\left.\partial^{2} P_{m}(x, \xi) / \partial \xi_{0}^{2}<0\right\}$ the set of the double characteristics of $P_{m}(x, \xi)$, we assume that $\Sigma_{2}$ is a $C^{\infty}$ manifold such that the canonical 1 -form $\omega$ does not vanish on $T \sum_{2}$.

(iii) $\forall \rho \in \Sigma_{2}$ denote by $V_{i \lambda}(\rho)$ the eigenspace corresponding to the purely imaginary eigenvalue $i \lambda$ of the fundamental matrix $F_{P_{m}}(\rho)$ of $P_{m}$ at $\rho$. Then,

$$
\operatorname{dim} \bigoplus_{\substack{i \lambda \neq 0 \\ i \lambda \in \operatorname{sp}\left(F_{P_{m}}(\rho)\right)}} V_{i \lambda}(\rho)=\text { const. }
$$

(iv) $P_{m}(x, \xi)$ vanishes exactly of order two on $\Sigma_{2}$.

The second assumption deals with the degeneracy of the real eigenvalues of $F_{P_{m}}(\rho)$ when $\rho$ is close to some critical subset $\sum_{2}^{\prime}$ of $\sum_{2}$.

(H2) There exists a subset of $\Sigma_{2}, \Sigma_{2}^{\prime}$, such that:

(i) $F_{P_{m}}(\rho)$ has a strictly positive eigenvalue, $\lambda(\rho)$ if and only if $\rho \in \Sigma_{2} \backslash \Sigma_{2}^{\prime}$.

(ii) Let

$$
r(\rho)=\operatorname{rank} F_{P_{m}}^{2}(\rho),
$$
when $\rho \in \Sigma_{2} \backslash \sum_{2}^{\prime}$, and

$$
r^{\prime}(\rho)=\operatorname{rank} F_{P_{m}}^{2}(\rho),
$$

when $\rho \in \sum_{2}^{\prime}$. Then $r(\rho) \equiv r, \forall \rho \in$ $\Sigma_{2} \backslash \Sigma_{2}^{\prime}, r^{\prime}(\rho) \equiv r^{\prime}, \forall \rho \in \Sigma_{2}^{\prime} \quad$ and moreover $r=r^{\prime}+2$.

(iii) The function $\lambda$ defined in (i) is (at least) a $C^{(1)}$ function difined on $\Sigma_{2}$. In particular this implies that $\lambda(\rho)$ $\leqslant \mathrm{d}_{\Sigma_{2}}^{2}\left(\rho, \Sigma_{2}^{\prime}\right)$ where $\mathrm{d}_{\Sigma_{2}}\left(\cdot, \Sigma_{2}^{\prime}\right)$ denotes any geodesic distance in $\Sigma_{2}$ from the set $\sum_{2}^{\prime}$.

Assumption (H2) has essentially a "static" nature, i.e. everything is looked at on a fixed point $\rho \in$ $\Sigma_{2}$. We also need a more "dynamical" type of assumption, which will be stated in (H3). To this end let us define what we mean by factorization of the principal symbol $P_{m}$. Without loss of generality we may assume in the following that $m=2$.

Definition 2.1. We say that $P_{2}(x, \xi)$ is factorized (in the sense of Ivrii [6]) if we can find $C^{\infty}$ symbols $l\left(x, \xi^{\prime}\right), m\left(x, \xi^{\prime}\right)$ vanishing on $\sum_{2}$, homogeneous of degree one, $q\left(x, \xi^{\prime}\right) \geq 0$ vanishing on $\Sigma_{2}$ and homogeneous of degree two, such that, denoting by $\Lambda(x, \xi)=\xi_{0}-l\left(x, \xi^{\prime}\right), M(x, \xi)=$ $\xi_{0}-m\left(x, \xi^{\prime}\right)$, we have:
(i ) $H_{\Lambda}(\rho) \in \operatorname{ker} F_{P_{2}}(\rho) \cap \operatorname{ran} F_{P_{2}}(\rho), \forall_{\rho} \in$ $\Sigma_{2}^{\prime}$.
(ii) $P_{2}(x, \xi)=-\Lambda(x, \xi) M(x, \xi)+q\left(x, \xi^{\prime}\right)$. 
(iii) $\{\Lambda, M\}(\rho)=0, \forall \rho \in \Sigma_{2}$.

(iv) $\forall \rho \in \Sigma_{2} \backslash \sum_{2}^{\prime}$ there exist $U_{\rho}$ conic neighborhood of $\rho$, a constant, $C_{\rho}>0$, such that:

$\left|\{\Lambda, q\}\left(p^{\prime}\right)\right| \leq C_{\rho} \sqrt{q}\left(\rho^{\prime}\right)$,

$\rho^{\prime} \in U_{\rho}$ and $C_{\rho} \leq c \lambda(\rho)$ for a suitable posi. tive constant $c$.

( v ) $H_{\Lambda}(\rho)-H_{M}(\rho) \in \operatorname{ker} F_{P_{2}}(\rho) \cap \operatorname{ran} F_{P_{2}}(\rho)$, $\forall \rho \in \sum_{2}$.

(H3) Assume that $P_{2}(x, \xi)$ is factorized according to Definition 2.1. Then there exists a homogeneous symbol of degree zero $f\left(x, \xi^{\prime}\right)$ vanishing on $\Sigma_{2}$ such that:

(i ) $\{\Lambda, f\}(\rho) \sim \lambda(\rho), \forall \rho \in \Sigma_{2}$.

(ii) $0 \leq\{\Lambda, f\}\left(x, \xi^{\prime}\right) \leq c_{1}\{M, f\}\left(x, \xi^{\prime}\right)$, for a suitable positive constant $c_{1}$.

(iii) These exists $\kappa \in$ ]0, 1 [ such that $\{q, f\}^{2}\left(x, \xi^{\prime}\right)$ $\leq 4 \kappa[\Lambda, f\}\left(x, \xi^{\prime}\right)\{M, f\}\left(x, \xi^{\prime}\right) q\left(x, \xi^{\prime}\right)$, for any $\left(x, \xi^{\prime}\right) \in U_{\bar{\rho}}, \bar{\rho} \in \Sigma_{2}^{\prime}, U_{\bar{\rho}}$ conical neighborhood of $\bar{\rho}$.

The last hypothesis is concerned with the lower order terms of the operator $P$.

(H4) There exist two positive constants $C, \varepsilon$ such that, $\forall \rho \in \Sigma_{2}$

$$
\begin{aligned}
\operatorname{dist} & \left(P_{1}^{s}(\rho),\left(\left[-\operatorname{Tr}^{+} F_{P_{m}}(\rho)+\varepsilon, \operatorname{Tr}^{+} F_{P_{m}}(\rho)-\varepsilon\right]\right.\right. \\
\leq C \lambda(\rho) & \times\{0\}) \cup\{(0,0)\}) \\
\leq &
\end{aligned}
$$

where dist denotes any distance in $\boldsymbol{C}$.

If $\operatorname{Tr}^{+} F_{P_{m}}(\rho)>0, \forall \rho \in \Sigma_{2}$, then the above condition becomes:

(a) $\operatorname{dist}\left(P_{1}^{s}(\rho),\left(\left[-\operatorname{Tr}^{+} F_{P_{m}}(\rho)+\varepsilon, \operatorname{Tr}^{+} F_{P_{m}}(\rho)\right.\right.\right.$ $-\varepsilon] \times\{0\}))<C \lambda(\rho)$.

On the other hand if $\operatorname{Tr}^{+} F_{P_{m}}(\rho) \equiv 0$ on $\Sigma_{2}$, then we obtain

(b) $\quad \operatorname{dist}\left(P_{1}^{s}(\rho),\{0,0\}\right) \leq C \lambda(\rho)$,

i.e. $\left|P_{1}^{s}(\rho)\right| \leq C \lambda(\rho)$. In the non effectively hyperbolic case, i.e. $\lambda_{\mid \Sigma_{2}} \equiv 0$, condition (a) becomes

$\operatorname{Im} P_{1}^{s}(\rho)=0, \quad-\operatorname{Tr}^{+} F_{P_{m}}(\rho)+\varepsilon \leq \operatorname{Re} P_{1}^{s}(\rho)$ $\leq \operatorname{Tr}^{+} F_{P_{m}}(\rho)-\varepsilon$,

while condition (b) means $P_{1}^{s}(\rho)=0$, thus reducing to the well-known Ivrii-Petkov-Hörmander Levi conditions ([9], [4], [6]).

We are now in a position to state the main theorem:

Theorem 2.1. Let $P$ be a second order differential operator verifying Assumptions (H1)-(H4). Then the Cauchy problem for $\boldsymbol{P}$ in $\Omega_{t}, t \in \boldsymbol{R}$ is well-posed in $C^{\infty}$.

3. Comments. In this Section we gather a number of results characterizing our geometrical situation as well as the different possible types of degeneracy which may occur. Due to Assumption (H1) (iv), denoting by $\bar{\rho}$ a point in $\Sigma_{2}^{\prime}$ and $V_{\bar{\rho}}$ a conical neighborhood of $\bar{\rho}$ we can find real valued symbols homogeneous of degree one, $\varphi_{j}\left(x, \xi^{\prime}\right), j=1, \ldots, k$, defined in $V_{\bar{\rho}}$ such that possibly after a conjugation with a Fourier integral operator leaving the time axis invariant $P_{2}$ becomes

$$
P_{2}(x, \xi)=-\xi_{0}^{2}+\sum_{j=1}^{k} \varphi_{j}^{2}\left(x, \xi^{\prime}\right),(x, \xi) \in V_{\bar{\rho}} .
$$

The following Proposition is essentially well known and we include it for a better understanding of the examples below.

Proposition 3.1. Let $P_{2}$ be as in 3.1 above and $\rho \in \Sigma_{2}$. Put:

$$
\begin{gathered}
v_{\rho}=\left(\left\{\xi_{0}, \varphi_{1}\right\}(\rho), \cdots,\left\{\xi_{0}, \varphi_{k}\right\}(\rho)\right), \\
M_{\rho}=\left[\begin{array}{cccc}
0 & \left\{\varphi_{1}, \varphi_{2}\right\}(\rho) & \cdots & \left\{\varphi_{1}, \varphi_{k}\right\}(\rho) \\
\left\{\varphi_{2}, \varphi_{1}\right\}(\rho) & 0 & \cdots & \left\{\varphi_{2}, \varphi_{k}\right\}(\rho) \\
\vdots & \vdots & \ddots & \vdots \\
\left\{\varphi_{k}, \varphi_{1}\right\}(\rho) & \left\{\varphi_{k}, \varphi_{2}\right\}(\rho) & \cdots & 0
\end{array}\right],
\end{gathered}
$$

and denote by $\pi_{\rho}: \boldsymbol{R}^{k} \rightarrow \operatorname{ker} M_{\rho}$ the projection operator onto $\operatorname{Ker} M_{\rho}$. Then either

a) $\pi_{0} v_{\rho} \neq 0$; in this case $P_{2}$ is effectively hyperbolic at $\rho \in \Sigma_{2}$.

or

b) $\pi_{\rho} v_{\rho}=0$; in this case the following situations occur:

( i ) $\left|M_{\rho}^{-1} v_{\rho}\right|>1$; then $F_{P_{2}}(\rho)$ has two real nonzero eigenvalues.

(ii) $\left|M_{\rho}^{-1} v_{\rho}\right|=1$; then $\operatorname{ker} F_{P_{2}}^{2}(\rho) \cap \operatorname{ran} F_{P_{2}}^{2}(\rho)$ $\neq\{0\}$, i.e. $F_{P_{2}}(\rho)$ has a Jordan block of size 4 in its canonical form.

(iii) $\left|M_{\rho}^{-1} v_{\rho}\right|<1$; then $\operatorname{ker} F_{P_{2}}^{2}(\rho) \cap \operatorname{ran} F_{P_{2}}^{2}(\rho)$ $=\{0\}$, i.e. $F_{P_{2}}(\rho)$ is non effectively hyperbolic.

Here $M_{\rho}^{-1} v_{\rho}$ means the unique vector belonging to $\left(\operatorname{ker} M_{\rho}\right)^{\perp}$ which is mapped to $v_{\rho}$ by $M_{\rho}$.

The next proposition asserts that there are no Jordan blocks of size 4 in the canonical form of $F_{P_{2}}(\rho)$.

Proposition 3.2. Assume that (H1), (H2) hold. Then $\forall \rho \in \Sigma_{2}^{\prime}$,

$\operatorname{ker} F_{P_{2}}^{2}(\rho) \cap \operatorname{ran} F_{P_{2}}^{2}(\rho)=\{0\}$.

Moreover if $\rho \in \sum_{2} \backslash \sum_{2}^{\prime}, \pi_{\rho} v_{\rho} \neq 0$.

Proposition 3.3. Assume that (H1), (H2) hold; let $\bar{\rho} \in \sum_{2}^{\prime}$ and $U_{\bar{\rho}}$ a conical neighborhood of $\bar{\rho}$; it is then possible to find a vector

$$
\gamma\left(x, \xi^{\prime}\right)=\left(\gamma_{1}\left(x, \xi^{\prime}\right), \ldots, \gamma_{k-\nu}\left(x, \xi^{\prime}\right)\right) \text {, }
$$


where $\gamma_{j}\left(x, \xi^{\prime}\right)$ 's are real symbols homogeneous of degree zero smoothly dependent on $x_{0}$, such that

(i) If

$$
\begin{aligned}
& \Lambda(x, \xi)=\xi_{0}+\left\langle\gamma\left(x, \xi^{\prime}\right), \varphi^{\prime \prime}\left(x, \xi^{\prime}\right)\right\rangle, \\
& M(x, \xi)=\xi_{0}-\left\langle\gamma\left(x, \xi^{\prime}\right), \varphi^{\prime \prime}\left(x, \xi^{\prime}\right)\right\rangle, \\
& Q\left(x, \xi^{\prime}\right)=\left|\varphi^{\prime}\left(x, \xi^{\prime}\right)\right|^{2}+ \\
& \left\langle(\operatorname{Id}-\gamma \otimes \gamma) \varphi^{\prime \prime}\left(x, \xi^{\prime}\right), \varphi^{\prime \prime}\left(x, \xi^{\prime}\right)\right\rangle,
\end{aligned}
$$

then

$$
P_{2}(x, \xi)=-\Lambda M+Q \text {; }
$$

(ii) $|\gamma(\rho)|<1$, for every $\rho \in \Sigma_{2} \cap U_{\bar{\rho}}$;

(iii) $\{\Lambda, Q\} \leq C\left(Q+\left|\partial_{0} \varphi^{\prime}\right| \sqrt{Q}\right)$;

(iv) $\{\Lambda, M\}(\rho)=0$, for every $\rho \in \Sigma_{2} \cap U_{\bar{\rho}}$.

Proposition 3.4. Assume (H1), (H2) hold. Furthermore suppose that if $\forall \bar{\rho} \in \sum_{2}^{\prime}$ there exists a closed, proper cone $\Gamma(\bar{\rho}) \subset T_{\bar{\rho}} T^{*} \Omega$ such that

$$
\lim _{\substack{\rho \rightarrow \bar{\rho} \\ \rho \in \Sigma_{2} \backslash \Sigma_{2}^{\prime}}} z_{\rho} \in \Gamma(\bar{\rho}),
$$

where $z_{\rho}$ denotes the eigenvector corresponding to the positive eigenvalue $\lambda(\rho), \rho \in \Sigma_{2} \backslash \Sigma_{2}^{\prime}$, then condition (i) in Assumption (H3) can always be fulfilled.

Remark 3.1. If the manifold $\sum_{2}^{\prime}$ is cylindrical with respect to $x_{0}$, then $\Lambda=M=\xi_{0}$ yields a factorization in the sense of Definition 2.1, and condi. tion (iii) of (H3) becomes:

$\left\{\xi_{0}, f\right\}^{2}(x, \xi) P_{2}(x, \xi)+C\left\{P_{2}, f\right\}^{2}(x, \xi) \geq 0$, for a suitable positive constant $C$, in a neighborhood of a point $\bar{\rho} \in \sum_{2}^{\prime}$.

We now exhibit a number of examples and counterexamples to our Assumptions (H1)-(H4). Let us start first with some models which do not satisfy at least one of the above mentioned assumptions.

A. Let $P_{2}(x, \xi)=-\xi_{0}^{2}+\left(x_{0} x_{2}^{2}-x_{1}\right)^{2} \xi_{n}^{2}+$ $r \xi_{1}^{2}$, near $\left(0, e_{n}\right), r>0$. Using the notations of Proposition 3.1 we have

$$
\begin{aligned}
& v=\left(x_{2}^{2}, 0\right) \text { and } M=\left[\begin{array}{cc}
0 & \sqrt{r} \\
-\sqrt{r} & 0
\end{array}\right], \\
& \text { hence } \pi v=0 \text { and } M^{-1} v=\left(0, \frac{x_{2}^{2}}{\sqrt{r}}\right) \text { which }
\end{aligned}
$$

can coincide with one of the cases of item b) in Proposition 3.1 according to $x_{2}^{2}<\sqrt{r}, x_{2}^{2}$ $=\sqrt{r}$ or $x_{2}^{2}>\sqrt{r}$. Therefore Assumption (H2) is not satisfied.

B. Let

$$
\begin{gathered}
P_{2}(x, \xi)=-\xi_{0}^{2}+\left(x_{0}\left(x_{2}^{2}-x_{3}^{2}\right)-x_{1}\right)^{2} \xi_{n}^{2}+ \\
\left(2 b x_{2} x_{3} x_{0}-x_{4}\right)^{2} \xi_{n}^{2},
\end{gathered}
$$

near $\left(0, e_{n}\right), b \in \boldsymbol{R}$. It is easy to show that Hypotheses (H1), (H2) are verified and that $\lambda \sim x_{2}^{2}+x_{3}^{2}$. Moreover $\Lambda(x, \xi)=M(x, \xi)$ $=\xi_{0}$ yields a factorization in the sense of
Definition 2.1. On the other hand (i) of (H3) is not satisfied: there is no choice of $C^{\infty}$ functions $\alpha, \beta$ such that

$$
\begin{array}{r}
\partial_{0}\left[\alpha\left(x_{0}\left(x_{2}^{2}-x_{3}^{2}\right)-x_{1}\right)+\right. \\
\left.\beta\left(2 b x_{0} x_{2} x_{3}-x_{4}\right)\right] \geq 0,
\end{array}
$$

since it is easily verified that there is no closed, proper cone $\Gamma(\bar{\rho}), \bar{\rho} \in \sum_{2}^{\prime}$, satisfying condition (3.2) of Proposition 3.4.

C. Let $P_{2}(x, \xi)=-\xi_{0}^{2}+\left(x_{0} x_{2}^{2 l}-x_{1}\right)^{2} \xi_{n}^{2}+$ $k_{1} \xi_{n}+i k_{2} x_{2}^{l} \xi_{n}$, near $\left(0, e_{n}\right)$, where $k_{1}>0$, $k_{2} \in \mathbf{R}, l \in \boldsymbol{N}$. This model satisfies all our assumptions (H1)-(H4). Analogously

$P_{2}(x, \xi)=-\xi_{0}^{2}+\left(x_{0}\left(x_{2}^{2}+\xi_{2}^{2} \xi_{n}^{-2}\right)-x_{1}\right)^{2} \xi_{n}^{2}+$ $\left(x_{0} x_{3}^{2}-x_{4}\right)^{2} \xi_{n}^{2}+k_{1}\left(x, \xi^{\prime}\right)$,

near $\left(0, e_{n}\right)$, where

$$
\begin{aligned}
\operatorname{Im} k_{1}\left(x, \xi^{\prime}\right)=O\left(x_{2}^{2}+\xi_{2}^{2} \xi_{n}^{-2}+x_{3}^{2}\right), & \\
& \operatorname{Re} k_{1}\left(x, \xi^{\prime}\right)>0,
\end{aligned}
$$

satisfies (H1)-(H4).

D. Let $P_{2}(x, \xi)=-\xi_{0}^{2}+\left(x_{0} x_{2}^{2 l}-x_{1}\right)^{2} \xi_{n}^{2}+$ $k(x) \xi_{n}$, near $\left(0, e_{n}\right)$, where $k(x)$ is a smooth function. After a symplectic dilation in the variables $\left(x_{1}, \xi_{1}\right)$ we reduce $P_{2}$ to the following symbol

$$
\tilde{P}_{2}(x, \xi)=-\xi_{0}^{2}+x_{0}^{2} x_{2}^{4 l} \xi_{n}^{2}+\tilde{k}(x) \xi_{n} .
$$

Recalling the celebrated necessary conditions of Ivrii-Petkov ([9], [8]) we see that in order that the local Cauchy problem for $\tilde{P}_{2}$ (and hence for $P_{2}$ ) be well-posed we must necessarily have $\tilde{k}(x)=x_{2}^{2 l} \tilde{\tilde{k}}(x)$. Hence if $k_{1}$ in Example $\mathrm{C}$ is zero, we conclude that Assumption (H4) cannot be improved.

E. In order to give an idea of the microlocal energy estimate needed in the present situation we exhibit an elementary estimate for the operator in Example D. We will not enter into the details of the symbolic calculus and proceed only at a formal level.

Denote by $f^{2}(x)=\left(x_{0} x_{2}^{2 l}-x_{1}\right)^{2}+\xi_{n}^{-1}$; this is the formal time function used in the estimate. The operator $P_{2}$ satisfies all our assumptions and $f$ verifies the conditions of Hypothesis (H3). Denote by $\|v\|$ the $L^{2}$-norm of a $C_{0}^{\infty}$ function $v$ in the open set $\Omega$. Therefore we compute

$$
\begin{aligned}
& \text { (3.3) } 2 i \operatorname{Im}\left\langle f^{-N} P_{2} u,-f^{-N} D_{0} u\right\rangle \\
& =2 i \operatorname{Im}\left\langle f^{-N}\left(-D_{0}^{2}+f^{2} D_{n}^{2}+D_{n}+i k x_{2}^{2 l} D_{n}\right) u,\right. \\
& \left.\quad-f^{-N} D_{0} u\right\rangle
\end{aligned}
$$




$$
\begin{aligned}
& -2 i N \operatorname{Re}\left[\left\|f^{-N-1 / 2} x_{2}^{l} D_{0} u\right\|^{2}\right. \\
& \left.+\frac{N-1}{N}\left\|f^{-N-1 / 2} x_{2}^{l} D_{n} u\right\|^{2}+\left\|f^{-N-1 / 2} x_{2}^{l} D_{n}^{1 / 2} u\right\|^{2}\right] \\
& \quad-2 i \operatorname{Im}\left\langle f^{-N+1 / 2} i k x_{2}^{l} D_{n} u, f^{-N-1 / 2} x_{2}^{l} D_{0} u\right\rangle \\
& =D_{0} E_{1}-2 i N E_{2}+i \mathscr{R} . \\
& \quad \text { It is easy to see that }|R| \text { can be estimated }
\end{aligned}
$$
by $N E_{2}$ provided $N$ is sufficiently large and that $E_{1}$ and $E_{2}$ define positive energies. We also note how the positivity of the real eigenvalue of $F_{P_{2}}, \lambda=x_{2}^{2 l} \xi_{n}$, plays a crucial role in establishing the estimate.

Theorem 2.1. is a consequence of the following Proposition:

Proposition 3.5. The following (local) energy estimate holds:

$$
\begin{aligned}
\int_{-\infty}^{0} e^{-2 \tau x_{0}} & \|P u\|_{N+s}^{2} d x_{0} \\
& >C \tau^{2} \int_{-\infty}^{0} e^{-2 \tau x_{0}}\left[\|u\|_{s+\frac{1}{2}}^{2}+\left\|D_{0} u\right\|_{s}^{2}\right] d x_{0} \\
& -C_{l}^{\prime} \tau \int_{-\infty}^{0} e^{-2 \tau x_{0}}\left[\|u\|_{-l}^{2}+\left\|D_{0} u\right\|_{-l}^{2}\right] d x_{0},
\end{aligned}
$$

for every $\forall s \in \boldsymbol{R}, \forall l \in \boldsymbol{R}$.

Aknowledgements. The authors would like to thank T. Nishitani and S. Wakabayashi for helpful conversations. At an early stage of the preparation of this manuscript they could also profit of useful comments of V. Petkov and F. Trèves.

\section{References}

[1] E. Bernardi, A. Bove and C. Parenti: Geometric results for a class of hyperbolic operators with double characteristics. J. Func. Anal., 116, 62-82 (1993).

[ 2 ] L. Hörmander: Fourier integral operators. I. Acta Math., 127, 79-183 (1971).

[ 3 ] L. Hörmander: Quadratic hyperbolic operators (1992) (preprint).

[4] L. Hörmander: The Cauchy problem for differential equations with double characteristics. J. Analyse Math., 32, 118-196 (1977).

[5] L. Hörmander: The Analysis of Linear Partial Differential Operators. vols. 1-4, Springer-
Verlag, Berlin (1985).

[6] V. Ja. Ivrii: The well-posedness of the Cauchy problem for non-strictly hyperbolic operators. III. The Energy Integral. Trans. Moscow Math. Soc., 34, 149-168 (1978).

[7] V. Ja. Ivrii: Wave fronts of solutions of certain hyperbolic pseudodifferential equations. Trans. Moscow Math. Soc., 39, 87-119 (1979).

[ 8] V. Ja. Ivrii : Linear Hyperbolic Equations. Encyclopedia of Mathematical Sciences. Partial Differential Equations, vol. 4, 33, 149-235 (1993) (translated from Itogi Nauki i Tekniki, 33, VINITI, Moscow, 1988).

[9] V. Ja. Ivrii and V. M. Petkov: Necessary conditions for the Well-posedness of the Cauchy problem for non-strictly hyperbolic equations. Russian Math. Surveys, 29, 1-70 (1974).

[10] N. Iwasaki: The Cauchy problem for effectively hyperbolic equations (a Special Case). J. Math. Kyoto Univ. , 23, 503-562 (1983).

[11] K. Kajitani and S. Wakabayashi: Propagation of singularities for several classes of pseudodifferential operators. Bull. Sc. Math., $2^{\mathrm{e}}$ série, 115 397-449 (1991).

[12] K. Kajitani, T. Nishitani and S. Wakabayashi : The Cauchy problem for hyperbolic operators of strong type. Tsukuba University (1992) (preprint).

[13] B. Lascar: Propagation des singularités Gevrey. Am. J. Math., 110, 413-449 (1988).

[14] B. Lascar, R. Lascar and N. Lerner: Propagation of singularities for non-real pseudo-differential operators (1993) (preprint).

[15] R. B. Melrose: The Cauchy problem and propagation of singularities. Seminar on Nonlinear Partial Differential Equations (ed. S. S. Chern). vol. 2, Springer-Verlag, M.S.R.I. Publications, pp. 185-201 (1984).

[16] R. B. Melrose: The Cauchy problem for effectively hyperbolic operators. Hokkaido Math. J., 12, 371-391 (1983).

[17] T. Nishitani: The effectively hyperbolic Cauchy problem. Lect. Notes in Math., vol. 1505, pp. 71-167 (1991).

[18] T. Nishitani: Local energy integrals for effectively hyperbolic operators. I, II. J. Math. Kyoto Univ., 24, 623-658, 659-666 (1984). 
